\begin{tabular}{|c|c|c|c|c|c|}
\hline Revista Praxis & ISSN: $1657-4915$ & Vol. 13 & No. 2 & 181 - 198 & julio - diciembre de 2017 \\
\hline \multicolumn{6}{|c|}{ DOI: http://dx.doi.org/10.21676/23897856.2365 } \\
\hline
\end{tabular}

\title{
PERSPECTIVAS QUE HAN DIRECCIONADO LA INVESTIGACIÓN Y CONSOLIDACIÓN DE LA EDUCACIÓN EN CIENCIAS COMO UNA DISCIPLINA CIENTÍFICA
}

\author{
PERSPECTIVES THAT HAVE ADDRESSED THE RESEARCH AND CONSOLIDATION OF \\ SCIENCE EDUCATION AS A SCIENTIFIC DISCIPLINE
}

\author{
Boris Fernando Candela-Rodríguez ${ }^{1}$
}

\begin{abstract}
RESUMEN
A lo largo de la historia del campo de la educación en ciencias los investigadores han focalizado sus intereses de indagación, en dar respuesta a las diferentes problemáticas relacionadas con el aprendizaje y la enseñanza del currículo de las ciencias. Para ello, diseñaron y desarrollaron investigaciones, las cuales han estado informadas e iluminadas por los marcos teóricos provenientes de la psicología cognitiva y sociocultural. La anterior situación produjo en este campo las tradiciones de investigación del cambio conceptual y perspectiva sociocultural. Estas han delineado los problemas, los marcos teóricos y las metodologías de investigación que ayudaron a los investigadores a desarrollar y consolidar el campo de la educación en ciencias como una disciplina científica. Por tanto, este documento tuvo como propósito central la descripción de manera sucinta de las trayectorias epistémicas recorridas por los miembros de esta comunidad de práctica, con el fin de que pueda ser utilizada por los investigadores noveles en su proceso de contextualización.
\end{abstract}

Palabras clave: Educación en ciencias; Psicología cognitiva; Psicología sociocultural; Tradiciones de investigación.

\begin{abstract}
Throughout the history of the field of science education researchers have focused their interests of inquiry, in responding to the different problems related to learning and teaching the science curriculum. For this, they designed and developed research, which has been informed and illuminated by the theoretical frameworks from cognitive and sociocultural psychology. This situation produced in this field the research traditions of conceptual change and sociocultural perspective. These have delineated the problems, the theoretical frameworks and the research methodologies that helped researchers to develop and consolidate the field of science education as a scientific discipline. Therefore, this document had as its central purpose the description succinctly of the epistemic trajectories covered by the members of this community of practice, so that it can be used by new researchers in their process of contextualization.
\end{abstract}

Keywords: Science Education; Cognitive Psychology; Sociocultural Psychology; Research Traditions.

Fecha de recepción: enero 18 de 2017 / Fecha de aceptación: septiembre 8 de 2017 / Publicado en línea: noviembre 15 de 2017 Tipología: Artículo de Reflexión

Para citar éste artículo: Candela-Rodríguez, B. (2017). Perspectivas que han direccionado la investigación y consolidación de la educación en ciencias como una disciplina científica. Praxis, 13(2), 177-194. Doi: http://dx.doi.org/10.21676/23897856.2365

1. Mg. Universidad del Valle, Cali, Colombia. Correo electrónico: bofeca65@yahoo.com ORCID: 0000-0002-5833-1975 


\section{INTRODUCCION}

A l interior de la comunidad de educación en ciencias se han generado algunos interrogantes que dan cuenta del proceso educativo que se vive en las aulas, entre los que se destacan: (a) ipor qué los estudiantes no logran aprender los contenidos de las ciencias los cuales son planeados y enseñados por sus profesores?, (b) ¿por qué aún persiste la enorme dificultad en el aprendizaje de dichos contenidos?, (c) ¿cómo asistir a los profesores en formación y ejercicio en la identificación y desarrollo del conocimiento pedagógico del contenido?, (d) ¿cómo diseñar ambientes de aprendizaje de contenidos específicos potenciados por las TIC?, (e) icuáles son las concepciones alternativas sobre un contenido particular con las que llegan los estudiantes al aula de ciencias?, entre otros.

Estos interrogantes han sido un referente problematizador para el desarrollo del marco teórico que subyace al campo de la educación en ciencias. El constructo campo de la misma, desde el contexto anglosajón, es conceptualizado como una disciplina científica que está configurada por problemas, metodologías, marcos teóricos, órganos de divulgación e instituciones que agremian a los investigadores en educación. El cuerpo teórico que sustenta a cada uno de estos elementos comenzó a ser desarrollado por la comunidad de investigadores en Educación en Ciencias desde 1930 (Abell y Lederman, 2007; Bybee, 1977; Fraser y Tobin, 1998; Fraser, Tobin y McRobbie, 2012; Gabel, 1994; Jenkins, 2000).

Con el propósito deliberado de dar respuesta a los anteriores interrogantes, los diferentes grupos de investigación adscritos a la comunidad de educación en ciencias, han generado en los últimos 40 años una extensa literatura, la cual ha tratado los problemas del campo desde diferentes perspectivas (Duit, 2007). Desde luego, los trabajos llevados a cabo por estos investigadores descansan sobre diferentes marcos teóricos acerca de los elementos clave de la educación en ciencias, y algunos de ellos son: naturaleza, propósitos y naturaleza de la enseñanza-aprendizaje de las ciencias
(Bandiera, Caravita, Torracca y Vicentini, 1999; Duit, Gropengießer y Kattmann, 2005).

Conviene subrayar que los diferentes programas de investigación desarrollados al interior de esta comunidad no han seguido las mismas heurísticas de investigación. Esta situación se ha traducido en la producción de una literatura heterogénea que provoca una dispersión en la lectura y la interpretación de los diferentes informes y artículos que recogen la problemática en cuestión, en épocas disímiles. Ahora bien, una forma de generar un orden con el fin de ver el progreso en la literatura que representa las problemáticas de la enseñanza y el aprendizaje de las ciencias, es la de reconocer que al interior de esta comunidad de práctica existen comunidades de investigadores que comparten presupuestos teóricos y metodológicos afines.

En este sentido, es posible distinguir en la literatura sobre educación en ciencias (representad por medio de artículos, libros, informes de investigación, tesis de grado y Handbooks) cuatro programas de investigación destacados: a) la teoría conductista de Robert Gagne; b) la teoría del aprendizaje significativo de David Ausubel; (c) la teoría del desarrollo de Jean Piaget; y la teoría sociocultural de Vygotsky. Así, el cuerpo de conocimiento de estas teorías deja ver que son las precursoras de los diferentes paradigmas de investigación del campo. Por ejemplo, los presupuestos provenientes de la perspectiva sociocultural de Vygtosky han influenciado el desarrollo de líneas de investigación como el cambio conceptual y la cognición situada, donde el contexto de transacción de significado y formas de significar juegan un papel determinante.

Por tanto, el campo de la educación en ciencias a lo largo de su desarrollo y consolidación ha generado una diversidad de líneas de investigación. Desde luego que este hecho se ha dado como respuesta a la necesidad de dar solución a diferentes problemáticas relacionadas con la enseñanza y el aprendizaje de las ciencias. De ahí que resulte pertinente abordar de manera sucinta algunas de las perspectivas de indagación que han ejercido una fuerte influencia en el campo de la educación en ciencias. 


\section{TENDENCIAS DE LA INVESTIGACIÓN EN EDUCACIÓN EN CIENCIAS GENERADAS DESDE LAS PERSPECTIVAS DE PIAGET, AUSUBEL Y VYGOTSKY}

Conviene subrayar que los marcos teóricos y metodológicos construidos por Piaget, Ausubel y Vygotsky han sustentado el desarrollo de estudios de gran importancia para la enseñanza y el aprendizaje de las ciencias. Por tanto, dichos marcos les brindaron la oportunidad a los investigadores de este campo de moverse desde problemáticas educativas de carácter general, a indagaciones centradas en un dominio específico de las ciencias. Por esto, se considera pertinente representar de manera sucinta los principales aspectos que han tomado los investigadores del campo de la educación en ciencias desde estas tres perspectivas.

\section{Desde Piaget, pasando por Ausubel y llegando a Vygotsky}

Los marcos teóricos producidos por Piaget han influenciado diferentes estudios que recogen la problemática de la enseñanza y el aprendizaje de los contenidos de las ciencias en la escuela primaria y secundaria. Esta influencia fue particularmente clave en los setenta y ochenta. Un indicio claro de ello se aprecia en el número de citas sobre Piaget en los artículos publicados en las revistas de educación en ciencias a lo largo de este período (Anderson, 2007).

Conviene subrayar que el principal interés de Piaget por el aprendizaje no fue de tipo psicológico, sino epistemológico, pues Piaget pretendió desarrollar la epistemología desde una empresa meramente filosófica para un dominio empírico. Por consiguiente, se vio como un epistemólogo empírico que estuvo interesado en el desarrollo del conocimiento en los sujetos, de manera que su teoría fue denominada constructivismo (von Glasersfeld, 1995). Adicionalmente, la formación original de Piaget en la biología influenció su visión acerca de la construcción del conocimiento, que representó a través de la analogía de la adaptación de los seres vivos a sus ambientes. Esta orientación llegó a ser más obvia en su distinción entre la asimilación y la acomodación, junto con la idea de equilibración, las cuales jugaron un papel clave en el pensamiento de este epistemólogo.

Adicionalmente, Piaget describió el aprendizaje como un proceso interactivo en el cual un individuo le da sentido al mundo a través de sus esquemas cognitivos, que al mismo tiempo son desarrollados como un resultado de las acciones de los sujetos sobre los objetos del mundo. El anterior modelo fue acuñado por él a través de la siguiente frase: la inteligencia organiza el mundo y a su vez se organiza ella misma.

Por otro lado, Piaget consideró que el contexto social del estudiante juega un papel importante para el desarrollo del conocimiento al afirmar que la sociedad es la unidad suprema y los sujetos pueden lograr sus inventos y construcciones intelectuales, en la medida en que están en continuas interacciones dentro de un contexto de transacciones (Piaget, 1971). Sin embargo, en la mayoría de los escritos de Piaget y los artículos que direccionan el significado de su teoría en la educación en ciencias, el conocimiento fue descrito como una variedad de esquemas cognitivos en la cabeza de los sujetos, de ahí que esta teoría no destaca de manera explícita el elemento social como eje en la construcción del conocimiento.

Los investigadores del campo de la educación en ciencias adaptaron y ajustaron el marco teórico de Piaget con la intención de resolver sus propias problemáticas (Anderson, 2007; Duit, 2007; Duit, Gropengießer y Kattmann, 2005). Para ello, utilizaron el modelo de la adaptación que implica los procesos de asimilación y acomodación (Piaget, 1971). Así pues, la asimilación es conceptualizada como el elemento que le permite a un individuo interpretar la información sensorial, para luego incorporarla a su estructura cognitiva; mientras que la acomodación es el proceso mediante el cual la estructura cognitiva adopta un orden para darle sentido a una información específica del mundo natural, dado que la nueva información que el sujeto está incorporando no se ha logrado ajustar con la que ya posee. 
Los procesos de asimilación y acomodación no pueden estar disociados dentro del mecanismo de adaptación, sino que coexisten para que los sujetos puedan predecir y controlar su entorno. Ahora bien, si la información que está incorporando el individuo no se ajusta a la ya existente, se produce un conflicto cognitivo. Luego, el equilibrio puede ser restablecido por un proceso al que Piaget llamó equilibración, que es la interacción entre la asimilación y la acomodación.

Definitivamente, resulta fácil encontrar los elementos claves de la teoría de Piaget en la mayoría de los métodos constructivistas formulados en el campo de la educación en ciencias. De esto se infiere que esta perspectiva ha sido la piedra angular que funda muchas de las estrategias de enseñanza más utilizadas dentro del campo de la educación. Por ejemplo, el "Ciclo de Aprendizaje” (Véase Karplus, 1980) se fundamentó en la epistemología piagetiana, con el fin de asistir a los estudiantes en la construcción de una comprensión sobre un fenómeno natural específico.

A su vez, la perspectiva de Piaget vinculó la construcción del conocimiento por parte del sujeto con la teoría de los estadios de desarrollo. Así pues, propuso cuatro especies de operaciones lógicas que los niños y los adolescentes exhiben de manera secuencial, a saber: sensoriomotor (a los 18 meses de vida), preoperacional (hasta los siete años), operacional concreto (después de los siete años), y operacional formal (entre los once y quince años). Hay que destacar que no existe duda acerca de que la idea de las habilidades de pensamiento lógico general y su desarrollo en ciertas etapas juegan un papel importante durante la descripción del desarrollo cognitivo; sin embargo, hay un número de dificultades con la teoría de los estadios de Piaget.

Por ejemplo, la creencia de que las operaciones del pensamiento lógico son independientes del contexto ha sido seriamente problematizada. De hecho, la investigación ha mostrado que existe un fuerte efecto específico del dominio, es decir, la selección del estudiante sobre las operaciones lógicas depende del contenido particular de las ciencias y del contexto problemático. Por tanto, numerosos estudios han dejado ver que, a pesar de que las operaciones lógicas generales juegan un papel clave en el aprendizaje de las ciencias, estas no son universalmente transferibles a distintas tareas una vez se han aplicado en otras situaciones (Lawson y Thompson, 1988; Shayer y Wylam, 1981).

Por todo esto, se afirma que el trabajo de Piaget ha influenciado las perspectivas sobre la línea de investigación de las concepciones de los estudiantes y el aprendizaje conceptual en varias formas. Desde luego, sus relatos de cómo los sujetos llegan a aprender pueden ser vistos en muchos escritos de las concepciones de los estudiantes, así como pueden ser vistas las problemáticas del cambio conceptual y el constructivismo personal a través de las referencias sobre la asimilación y la acomodación. Adicionalmente, los métodos que Piaget utilizó para investigar la comprensión de los individuos, los cuales implicaron un entrevistador haciéndole interrogantes a los niños sin intentar inducir sus respuestas, han sido también usados en los estudios sobre las concepciones alternativas de los aprendices. También, la teoría de los estadios de Piaget ha servido para informar los diseños curriculares de las ciencias (ej., Science Curriculum Impromevement Study in the United States \& Cognitive Acceleration through Science Education in Britain (Adey y Shayer, 1993)).

Por otro lado, la teoría formulada por Piaget influyó en el desarrollo de la denominada psicología educativa de Ausubel, Novak y Hanesian (2005), quienes consideran que esta juega un papel clave para que los educadores de profesores, enseñantes de la escuela, puedan dar sentido a los múltiples fenómenos del aprendizaje de disciplinas específicas. Por tanto, ellos argumentaron que la mayor influencia en el desarrollo conceptual de los aprendices se encuentra en el conocimiento conceptual con el que llegan al aula para enfrentar el aprendizaje de los nuevos contenidos. De ahí que Ausubel et al. (2005) hayan acuñado la siguiente frase: "El factor más importante que influye en el aprendizaje es lo que el alumno ya sabe. Averígüese esto, y enséñese consecuentemente” (p.1). 
Así pues, uno de los aportes del marco teórico propuesto por Ausubel et al. (2005) a la investigación educativa ha sido la teoría del aprendizaje significativo, la cual t iene como elementos fundamentales la diferenciación progresiva, la reconciliación integradora y el organizador previo. Estos tres principios explican los procesos de razonamiento que le posibilitan al estudiante organizar los conceptos de la disciplina en cuestión, dentro de la estructura cognoscitiva que ya posee.

En este sentido, la diferenciación progresiva es el proceso a través del cual el sujeto organiza el nuevo conocimiento dentro de su estructura cognoscitiva de manera jerárquica, es decir, procediendo de arriba hacia abajo en términos de nivel de abstracción, generalidad e inclusividad. Para ello debe llevar a cabo una reconciliación integradora a partir del establecimiento del conjunto de similitudes y diferencias existentes entre el conocimiento que ya posee y el nuevo conocimiento que se le está presentando.

Ahora bien, para que el proceso de diferenciación progresiva y reconciliación integradora sea efectivo, se deben diseñar e implementar ambientes de aprendizaje que tengan entre uno de sus elementos constitutivos un organizador previo (noticias, animación, simulador, demostración, laboratorio, video clip, entre otros). Desde luego, este tiene como fin central permitirle al sujeto establecer un puente entre el conocimiento nuevo y el que ya existe en su mente. Así, el contenido de este instrumento debe tener un nivel de mayor generalidad, abstracción e inclusividad con respecto a la tarea de aprendizaje que se va abordar. Como lo afirman Ausubel et al. (2005), "este dispositivo pedagógico suministra un armazón o afianzamiento ideacional para la tarea de aprendizaje, además aumenta la discriminabilidad entre las nuevas ideas que van a ser aprendidas y las ideas relacionadas en la estructura cognoscitiva” (p.158).

Por otra parte, en los inicios de la década del setenta, un número de estudios empíricos fueron conducidos y focalizados en el aprendizaje de las ciencias en términos de factores específicos en un dominio de conocimiento, en lugar de tratar de comprender este proceso a partir de las habilidades de razonamiento general (ej., Driver, 1973; Viennot, 1979). De manera que es importante destacar el trabajo hecho por Novak (1987), el cual se centró en estudiar el contenido del razonamiento de los estudiantes en un dominio específico o sus concepciones alternativas sobre un fenómeno natural. También resulta pertinente resaltar la fuerte influencia que han jugado en el estudio de las concepciones alternativas los siguientes libros: The Pupil as Scientist por Rosalind Driver (1983) y Learning in Science: The implications of Children's Science, editado por Roger Osborne y Peter Freyberg (1985).

Ahora bien, el movimiento de las concepciones alternativas ganó una fortaleza en el mundo como consecuencia de la serie de conferencias organizadas por Joe Novak en Cornell University (1987). Estas impulsaron el desarrollo de múltiples estudios alrededor del mundo cuyo núcleo de investigación fueron las concepciones con las que llegaban los estudiantes al aula de ciencias. Este hecho influyó en el desarrollo exponencial del número de artículos publicados en las revistas de educación en ciencias y del desarrollo de esta línea de investigación (Anderson, 2007; Duit, Gropengießer y Kattmann, 2005).

Por todo esto, se ha comenzado a considerar que los procesos de construcción de significados y formas de significar están mediados por un elemento social el cual juega un papel crítico en la socialización y la transformación de los sujetos. Duit y Treagust (1998) argumentan que, si bien, un sujeto tiene que construir su propio significado de una nueva idea, el proceso de generación de este siempre está encarnado en un escenario social particular del cual es parte. No obstante, el constructivismo que dominó la educación en ciencias a lo largo de la década del 80, descuidó el elemento social en los procesos de construcción de significados y formas de significar, y en lugar de esto realizó un sobre énfasis en la producción de sentidos como un aspecto individual.

En este sentido, el discurso alrededor de la psicología ha comenzado a cambiar su foco de discusión, alejándose de una posición puramente cognitiva en términos de esquemas de 
procesamiento, hacia una interacción dialógica de los individuos dentro de auténticos escenarios de comunicación. Así pues, el eje fundamental de esta nueva tendencia de la investigación en el campo de la educación en ciencias, ha sido el trabajo de Vygotsky quien considera que la intersubjetividad, la interpretación, la comprensión y el lenguaje, juegan un papel crítico a lo largo de la construcción del conocimiento al interior de las comunidades de aprendizaje.

En efecto, investigadores del campo de la educación en ciencias, tales como Lemke (1990) y Sutton (1992), adoptaron y ajustaron el marco teórico de referencia de Vygotsky con el propósito de estudiar la influencia que tiene el desarrollo de las habilidades lingüísticas en la comprensión de las entidades y procesos que subyacen a los fenómenos naturales. Evidentemente, ellos consideran que el lenguaje es un elemento constitutivo de las ciencias, el cual media el desarrollo del pensamiento en conjunción con el aprendizaje de los contenidos científicos.

\section{DOS TRADICIONES DE INVESTIGACIÓN QUE HAN CATALIZADO LA CONSOLIDACIÓN DEL CAMPO DE LA EDUCACIÓN EN CIENCIAS}

Los presupuestos provenientes de las teorías de Piaget, Ausubel y Vygotsky han sustentado teórica y metodológicamente las principales tradiciones de investigación que configuraron el campo de investigación de la educación en ciencias, a saber: la tradición del cambio conceptual y la tradición sociocultural. En efecto, cada una de estas ha aportado elementos teóricos y metodológicos los cuales han sido el punto de referencia para llevar a cabo una amplia variedad de estudios, cuyo propósito central es el de mejorar la enseñanza y el aprendizaje de las ciencias. Esta situación le permitió al campo de la educación en ciencias alcanzar un desarrollo y la consolidación como disciplina de carácter científico.

\section{Tradición del cambio conceptual}

Esta es la tradición con el mayor tiempo de duración y alta influencia para los estudios llevados a cabo dentro del campo de la educación en ciencias. El cambio conceptual ha llegado a ser un constructo que destaca los aspectos clave de las diferentes orientaciones de enseñanza constructivistas implementadas en el período comprendido entre 1980 y 1990.

La investigación sobre el cambio conceptual se generó cuando los investigadores de la educación en ciencias comenzaron a articular los métodos de Piaget con las ideas sobre el desarrollo histórico del conocimiento científico, sobre todo con las teorías de Kuhn (1970) y Toulmin (1972). Posner, Strike, Hewson y Gertzog (1982) recogieron estos pensamientos en un artículo seminal, sugiriendo que los aprendices individuales tenían una "ecología conceptual", semejante a la utilizada por Toulmin para describir las disciplinas científicas. Adicionalmente, se comprendió que el aprendizaje de los contenidos por parte de los estudiantes es similar al proceso complejo de cambio que se da en las teorías al interior de los programas de investigación.

Como se ha venido afirmando, esta tradición tiene su génesis en la investigación en educación en ciencias (Posner et al., 1982) y en la psicología del desarrollo (Carey, 1985; Vosniadou, 1994). En el primer caso, la teoría del cambio conceptual implica que las concepciones de los estudiantes necesitan ser cambiadas por las nuevas nociones de las ciencias; de hecho, este presupuesto fue el núcleo del trabajo de Posner et al. (1982). En tanto, en el segundo caso, las ideas intuitivas de los aprendices son consideradas el punto a partir del cual se construyen de manera progresiva unas ideas más elaboradas o dichas ideas coexisten con los modelos científicos alternativos construidos y activados por los sujetos, dependiendo de la tarea y el contexto de actuación.

Según Duit y Treagust (1998), el constructo de cambio conceptual quizás induce a un número de malas interpretaciones, entre las cuales se mantiene la idea de que las preconcepciones de los estudiantes tienen que ser reemplazadas por modelos científicos. No obstante, la investigación en la educación en ciencias ha demostrado que esta situación es imposible de alcanzar. De hecho, la mayoría de los estudios llevados a 
cabo en la década del setenta y primeros años del ochenta han mostrado que las ideas "viejas” quedan "vivas” en un contexto particular. Por consiguiente, lo mejor que se podría lograr es un cambio conceptual superficial (Chinn y Brewer, 1993) en el que las partes de la idea inicial se fusionan con las partes de la idea nueva para formar alguna especie de idea híbrida. Adicionalmente, se ha probado que muchas de las concepciones cotidianas de los estudiantes (por ejemplo, concepciones del color, la luz, el calor) son fructíferas y valiosas para predecir y controlar su entorno cotidiano. Desde luego, muchas de las personas adultas, incluso los científicos, suelen representar estas concepciones intuitivas cuando están en contextos cotidianos.

En este sentido, en las últimas décadas, los miembros de la tradición del cambio conceptual han sostenido que el propósito deliberado de la instrucción en ciencias no es el de reemplazar las visiones cotidianas, pero si el de brindar las oportunidades a los estudiantes para que tomen conciencia de que, en ciertos contextos, las concepciones científicas son muchas más fructíferas que sus propias concepciones. Por tanto, el anterior presupuesto da comienzo a una nueva teoría del aprendizaje de la ciencia reconocida como la cognición situada (Brown, Collins y Duit, 1989). Esta teoría se ha apoyado en la visión de la dependencia de las concepciones al contexto sociocultural (Hennessy, 1993).

En la perspectiva de la cognición situada, el aprendizaje es visto como un proceso de enculturación o participación en las prácticas organizadas socialmente, a través de las cuales las habilidades especiales son desarrolladas por los aprendices en el momento en el que se comprometen con su propio aprendizaje dentro de un contexto específico (Rogoff, 1990). De acuerdo con Collins, Brown y Newman (1989), el componente clave del proceso de aprendizaje incluye el modelar, el entrenar, el apoyar, el desvanecerse y el animar a los aprendices para reflexionar sobre sus propias estrategias de resolución de los problemas. Así, este aprendizaje orienta al aprendiz para llegar a estar implicado en prácticas auténticas de una comunidad de práctica. Por tanto, esta perspectiva suministra un marco teórico valioso para describir y comprender los resultados apreciables de la investigación, los cuales muestran que el cambio conceptual no es fácil de lograr y que además está limitado por los contextos particulares.

\section{Tradición sociocultural como una participación dentro de una comunidad de aprendizaje a través del discurso científico}

Al interior de la comunidad de educación en ciencias se ha comenzado a generar un conjunto de críticas contra la perspectiva del cambio conceptual que predominó en la década de los 80 y primeros años de los 90. Esta situación se ha dado como consecuencia de que se focalizó solamente en el aprendizaje individual y descuidó el papel clave que juegan los aspectos sociales durante la construcción de significados. Adicionalmente, consideró el conocimiento como una variedad de estructuras almacenadas en las mentes de los sujetos, que configuran los modelos mentales del mundo externo (MartinezVélez, 2013).

Con el propósito de comenzar a superar la anterior restricción, se ha desarrollado una perspectiva constructivista sociocultural, la cual no solo se ha fundamentado en las ideas fenomenológicas, sino también en el trabajo de Vygotsky (1986). Otras fuentes que han apoyado esta nueva perspectiva de enseñanza-aprendizaje son los estudios constructivistas sociales, cuyo centro ha sido la génesis del conocimiento al interior de las comunidades científicas (KnorrCetina, 1981).

Conviene subrayar que la perspectiva constructivista sociocultural que ha venido siendo empleada en la educación en ciencias, generó la teoría de aprendizaje de la cognición situada (Brown et al., 1989; Hennessy, 1993; Roth, 2001). Desde este enfoque, el aprendizaje de un tópico específico significa la transformación progresiva de un lenguaje social cotidiano a un lenguaje social científico, que lleva a cabo un sujeto dentro de un contexto de negociación de significados y formas de significar. En este sentido, esta teoría ha comenzado a ser considerada por los investigadores en educación en ciencias como 
un elemento clave de la endoculturación de los sujetos, ya que actúa como una herramienta de pensamiento y aprendizaje.

Por todo esto, los investigadores de la educación en ciencias han asumido que la perspectiva de la cognición situada es un enfoque instruccional fructífero, dado que le permite al aprendiz introducirse a la nueva cultura a través de la orientación del experto, que en este caso es el profesor de ciencias. Por tanto, la participación del estudiante, tratando de resolver las tareas problemas enmarcadas en una situación problemática dentro de una comunidad de aprendizaje, le brinda a él mismo la oportunidad para que de manera progresiva alcance una alfabetización científica que informe la toma de decisiones a nivel personal, local, nacional y global.

Por otra parte, las ideas del constructivismo social en los últimos tiempos han ganado una posición dentro de la comunidad de educación en ciencias. De hecho, existen intentos de emplear dichas ideas con la intención de superar las limitaciones de los métodos constructivistas convencionales. En este sentido, se han producido estudios que han explorado las fortalezas de la perspectiva del constructivismo social, para investigar y apoyar la construcción de significados y formas de significar al interior de una comunidad de aprendizaje. Por ejemplo, el trabajo de Roth (1995) sobre la experimentación abierta en la enseñanza de las ciencias y los estudios sobre el diseño colaborativo (McGinn, Roth, Boutonné y Woszczyna, 1995) podrían ser tomados como referencias.

En dichas investigaciones se tiene como foco el estudio de la construcción colaborativa del significado y el aprendizaje. Así, en este campo, el potencial de la perspectiva constructivista sociocultural radica en que el conocimiento es “distribuido" y “compartido", en oposición a comprenderse como una propiedad privada de los sujetos. Así mismo, la visión del aprendizaje de las ciencias como la transformación gradual de una cultura a otra, abre nuevos horizontes que parecen ir más allá de lograr un aprendizaje de las ciencias más eficiente.

\section{DIVERSIDAD DE INTERESES DE INVESTIGACIÓN EN LA EDUCACIÓN EN CIENCIAS: FACTOR CLAVE PARA LA CONSOLIDACIÓN COMO DISCIPLINA DEL CARÁCTER CIENTÍFICO}

La revisión sistemática de una serie de Handbooks de investigación en educación en ciencias, junto con un amplio espectro de revistas de investigación en esta área, permitió evidenciar que los grupos de investigación de referencia del campo en cuestión han abordado un amplio espectro de tópicos de indagación en los últimos treinta años (por ejemplo, enseñanza, aprendizaje, currículum en ciencias, cambio conceptual, naturaleza de las ciencias, diseño de ambientes de aprendizaje de contenidos específicos, integración de las Tecnologías de la Información y la Comunicación TIC, conocimiento pedagógico del contenido, historia y filosofía de las ciencias, entre otros) (Abell y Lederman, 2007; Fraser y Tobin, 1998; Fraser, Tobin y McRobbie, 2012; Gabel, 1994). Desde luego, estos documentos incluyen trabajos relacionados con profesores, estudiantes, escuelas, museos, textos escolares, tecnología educativa, TIC, pedagogía, currículum, evaluación y temas de género. De ahí que se entre a afirmar que el marco teórico que funda al campo de la educación en ciencias es amplio y complejo, pues recoge cada uno los aspectos que ayudan a configurar la enseñanza y el aprendizaje de las ciencias tanto en escenarios formales como informales.

Adicionalmente, se destaca que la gran mayoría de los estudios llevados a cabo en el campo de la educación en ciencias, ha tenido sus objetos y sujetos de indagación en escenarios como la escuela secundaria, descuidando un poco lo que tiene que ver con la educación primaria y terciaria. Sin embargo, en las últimas décadas, los investigadores en educación en ciencias han aumentado su deseo por comprender los procesos de enseñanza y aprendizaje del currículum de las ciencias en contextos de la escuela primaria, superior y en la educación de carácter no formal. De ahí que este sea considerado por esta comunidad como un elemento que ha ayudado al desarrollo de la identidad de la educación en ciencias como una disciplina científica. 
En cuanto a las perspectivas del diseño metodológico utilizado por los investigadores con el fin de darle respuesta a sus interrogantes de investigación, se afirma que ellos en un inicio usaron enfoques cuasi-experimentales, cuya heurística se caracterizó por la identificación y el control de variables con el propósito deliberado de hallar una relación de covarianza entre estas. Así pues, dicho enfoque metodológico lo subyace una racionalidad positivista donde la objetividad juega un papel crítico. Ahora bien, en el comienzo de la década de los ochenta, los miembros de esta comunidad de práctica comenzaron a conceptualizar la enseñanza como un proceso complejo e incierto, en el que resulta difícil el control de variables. Esta situación quizás los motivó a ellos a moverse hacia una perspectiva metodológica de carácter cualitativo e interpretativo, donde el significado de las acciones, los acontecimientos y los procesos de los agentes educativos son un factor importante para explicar muchos de los fenómenos de la escuela. En este sentido, a esta perspectiva la funda el marco de la comprensión humana donde el lenguaje y la subjetividad son elementos centrales para la indagación.

Actualmente, la comunidad del campo de la educación en ciencias ha comenzado a considerar que las dos perspectivas anteriores no son excluyentes como inicialmente se había pensado. Por el contrario, estas se pueden utilizar de forma complementaria (perspectiva mixta) para de esta manera alcanzarse un proceso de triangulación por método, el cual le brindaría confiabilidad y credibilidad a la serie de resultados y conclusiones provenientes de los estudios bajo consideración. Bajo este supuesto, actualmente varios estudios de investigación publicados han asumido el enfoque mixto de investigación (Gorard y Taylor, 2004).

Por otra parte, la investigación sobre la enseñanza y el aprendizaje de las ciencias tiene una rica historia, la cual ha sido dividida en varios programas de investigación: proceso-producto, tiempo y aprendizaje, cognición del alumno y mediación de la enseñanza, ecología del aula, proceso del aula y la investigación de la ciencia cognitiva, y cognición del profesor y la toma de decisiones (Shulman, 1987). Se destaca que cada uno de dichos programas asumió los marcos metodológicos de la perspectiva de investigación cuantitativa o cualitativa, con la intención de resolver los problemas formulados por el programa de investigación en cuestión. Por ejemplo, los estudios adscritos al proceso-producto utilizaron una metodología cuasi experimental, en tanto, los de la cognición del profesor y la toma de decisiones implementaron un enfoque metodológico interpretativo.

Las anteriores asunciones permiten considerar que la naturaleza del campo de la educación en ciencias está caracterizada por una marcada diferencia en los elementos conceptuales y metodológicos, además de la considerable diversidad en el foco de los tópicos de investigación. Por consiguiente, el cuerpo de conocimientos del campo en consideración deja ver en el mundo dos perspectivas de investigación sobre la enseñanza y el aprendizaje de las ciencias, a saber: pedagógico/curricular y empírica/teórica (Jenkins, 2000).

La tradición pedagógico/curricular tiene como foco de investigación mejorar la práctica educativa, es decir, la enseñanza de las ciencias. Desde luego que en el momento donde los estudiantes logren una comprensión conceptual e integral de las grandes ideas y prácticas de las ciencias, es a causa quizás de una enseñanza ejemplar de esta disciplina. Sin embargo, se ha evidenciado que muchos de los marcos teóricos provenientes de dichos estudios no han logrado impactar las aulas de ciencias, razón por la cual se afirma que el campo de la educación en ciencias está en un estado de crisis. Así pues, muchos de los estudios que llevan a cabo actualmente, tienen como propósito deliberado ayudar a superar dicha crisis a través de la articulación intencionada de la teoría con la práctica, desde los diferentes ámbitos que configuran la enseñanza y el aprendizaje del currículum de las ciencias.

La tradición pedagógico/curricular en términos investigativos fundamentó la gran mayoría de reformas curriculares en ciencias desde la década del sesenta. Por lo general, dicha tradición les entregó a los profesores consejos prácticos de cómo diseñar la enseñanza y gestionar el aula de 
ciencias. Adicionalmente, suministró materiales curriculares como textos escolares e implementos para demostraciones y prácticas de laboratorio.

Esta tradición de la investigación en educación en ciencias ha estado focalizada más en USA que en Europa. Su naturaleza investigativa se ha centrado en los estudios cuasi-experimentales enmarcados dentro de una racionalidad positivista, cuyo fin es el de obtener un conjunto de "datos objetivos" con el propósito de comprender e influir en una realidad educativa. Desde luego que esta también ha tenido como meta central mejorar los procesos de enseñanza y aprendizaje de las ciencias. En los últimos treinta años, como consecuencia de la conceptualización de la enseñanza y el aprendizaje como procesos complejos y sutiles, se ha dado un cambio en las perspectivas metodológicas desde lo cuantitativo a lo cualitativo.

Retomando, la revisión de la literatura que recoge el campo de la educación en ciencias ha dejado evidenciar que las dos tradiciones de investigación tienen la misma meta central: mejorar la calidad de la enseñanza para asistir con esta el aumento del aprendizaje por comprensión conceptual e integrada de los tópicos del currículum de las ciencias. Esta situación quizás ha llevado a pensar a los miembros de esta comunidad de práctica que los dos enfoques de investigación (cuantitativo y cualitativo) en lugar de ser excluyentes son complementarios. Así pues, en los actuales momentos muchos estudios de investigación han asumido como perspectiva metodológica la mixta.

Finalmente, el cuerpo de conocimientos que configura a los órganos de divulgación del campo de la educación en ciencias (revistas en educación en ciencias, Handbook de investigación, libros, entre otros), permite ver los siguientes presupuestos, los cuales son una evidencia de la manera como esta área ha sufrido una evolución progresiva:

1. Las diferentes contribuciones que los estudios de investigación en educación en ciencias ha hecho tanto a las políticas como a las prácticas educativas, deberían ser sometidas a una evaluación rigurosa, considerando que se ha demostrado que muchos de estos estudios carecen de sólidos diseños metodológicos de investigación, situación que se traduce en una ruptura entre la teoría generada al interior de los grupos de investigación, y las prácticas del diseño y la enseñanza de tópicos específicos.

2. El campo de la educación en ciencias tiene una historia desde el comienzo de 1900 , como lo dejan ver los varios Digests Curtis of Investigations on the Teaching of Science, que recogen la investigación en esta área en USA desde 1906 hasta 1957. Desde luego, este tipo de estudio fue de perspectiva cuantitativa y de corte positivista. Por tanto, la educación en ciencias no se origina en la década del sesenta y setenta, como lo afirman algunos educadores (Boenig, 1969; Jenkins, 2000) de profesores de ciencias.

El cuerpo teórico y metodológico del campo de la educación en ciencias se ha caracterizado por su amplia diversidad en elementos, tales como tópicos de investigación, perspectivas metodológicas, ubicación institucional o departamental de los investigadores, y antecedentes profesionales de estos.

\section{LAS REFORMAS CURRICULARES COMO EVIDENCIA DEL DESARROLLO Y CONSOLIDACIÓN DEL CAMPO DE LA EDUCACIÓN EN CIENCIAS}

En el tiempo del crecimiento y la estabilidad económica de algunos países occidentales durante la era de la guerra fría, los políticos, industriales y diseñadores de la educación comenzaron a mostrar interés por la calidad de la educación en ciencias. De ahí que tomaran la decisión de diseñar currículos alternativos a gran escala con el propósito de mejorar la enseñanza y el aprendizaje en las aulas. Quizás ellos consideraron que dicha situación se lograría traducir en un desarrollo científico y tecnológico para estos países.

Con el fin de alcanzar la meta presupuestada arriba, en USA se inició la ola del diseño de proyectos curriculares a gran escala, cuyos marcos teóricos y metodológicos, junto con los materiales instruccionales, influenciaron fuertemente 
las reformas en la educación en ciencias tanto en países americanos como europeos. Desde luego que el pensamiento innovador acerca de la educación en ciencias que fundamentó los movimientos de reformas curriculares fue inspirado por el conocimiento disciplinar, la sabiduría artesanal relacionada con la práctica de la enseñanza y las teorías educativas y psicológicas (Psillos, 1997).

En este sentido, Bybee (1977) afirma que la sociedad ha ejercido una fuerte influencia en los cambios de la educación en ciencias durante sus principales períodos de transformación. De ahí que considere que factores sociales, tales como los económicos, los tecnológicos, los industriales, los ambientales, los políticos, los éticos y los morales, determinan la manera como los educadores de profesores e investigadores perciben las nuevas formas de la educación en ciencias. Por tanto, Bybee declara que estas condiciones sociales son uno de los factores dinamizadores de las diferentes reformas curriculares que ha sufrido la educación en ciencias en el mundo.

Ahora bien, otro aspecto clave para el diseño y la implementación de las reformas curriculares llevadas a cabo principalmente en el contexto anglosajón, hace referencia a la evolución progresiva que ha sufrido el campo de la educación en ciencias. En efecto, a lo largo de sus 100 años de existencia, este muestra una serie de transformaciones de manera progresiva y evolutiva en elementos estructurales como sus ideales intelectuales, sus problemas, sus metas, sus variantes conceptuales y metodológicas, sus órganos de divulgación y sus foros de discusión. Naturalmente, la interacción bidireccional de estos factores es el caldo de cultivo a través del cual se configura la estructura básica de la educación en ciencias, la cual quizás ha venido informando e iluminando las diferentes reformas curriculares en ciencias.

Por otro lado, se considera que la educación en ciencias de la escuela primaria y secundaria tiene una historia de reformas curriculares, las cuales se han originado como consecuencia de las diferentes necesidades contextuales producidas dentro de los sistemas sociales de los países. Así pues, los políticos, gobernantes y diseñadores curriculares influenciados de manera explícita o implícita por los marcos teóricos de los investigadores en educación, han visto el currículum de ciencias como un vehículo para darle solución a las situaciones problemáticas de orden local, nacional y global con las que se enfrenta a diario la población en general.

En este sentido, resulta probable que, a lo largo de la historia del campo de la educación en ciencias, los miembros de esta comunidad de práctica, en conjunción con sus instituciones y órganos de divulgación, hayan intervenido de manera directa o indirecta en el diseño de las diferentes reformas curriculares del área en cuestión, ya sea por un interés particular o por encargo del gobierno de turno. Quizás a cada una de dichas reformas las subyacen algunos de los fines intelectuales establecidos de manera consensuada por los miembros de este campo de indagación, por ejemplo, formar científicos, alfabetizar científicamente a todos los ciudadanos y generar una fuerza laboral científicamente formada, entre otros.

Conviene subrayar que estos fines del campo de la educación en ciencias ayudaron a catalizar la formulación y la sustentación de un conjunto de problemas sobre la enseñanza y el aprendizaje de las ciencias. Así pues, los investigadores a lo largo de la existencia de dicho campo han construido de manera colegiada las soluciones a dichas situaciones problemáticas, las cuales constituyen los diferentes marcos teóricos que han venido direccionando una serie de reformas curriculares en muchos países.

Desde luego, para darle respuesta de manera selectiva y sistemática a cada una de las diferentes problemáticas formuladas al interior del campo de la educación en ciencias, sus miembros tuvieron que adherirse a una perspectiva metodológica. Naturalmente, en los primeros años del desarrollo de este campo, sus integrantes utilizaron perspectivas metodológicas de carácter cuantitativo y diseños cuasi-experimentales. Con el paso de los años, el enfoque metodológico de ellos se movió hacia una metodología de corte cualitativo donde la subjetividad juega 
un papel clave. En los actuales momentos, los investigadores han comenzado a considerar que estas dos perspectivas metodológicas, en lugar de ser excluyentes, son complementarias; de ahí que se haya iniciado la utilización de diseños metodológicos mixtos, los cuales quizás brindan confiabilidad a la serie de resultados obtenidos desde dichos estudios (Abell y Lederman, 2007).

Por tanto, resulta probable entrar a considerar que el marco teórico y metodológico que subyace a las reformas curriculares en la enseñanza de las ciencias, refleja de manera latente las genealogías escolásticas e institucionales por las que ha atravesado el campo de la educación en ciencias a lo largo de su evolución histórica. Así mismo, el diseño de estas series de reformas sirve de evidencia empírica para apoyar la tesis de que la educación en ciencias es una empresa racional e histórica, la cual ha alcanzado un desarrollo y consolidación como una disciplina científica, ya que la teoría generada desde esta ha servido como punto de referencia para resolver los problemas prácticos de la enseñanza y el aprendizaje de las ciencias. Sin embargo, a pesar de que a dichas reformas les subyace un rico marco teórico de perspectivas alternativas en educación, muchas de estas solo se han quedado en los currículos prescriptos por las políticas educativas sin lograr impactar las prácticas educativas en el aula, situación que, se reitera, ha sido asumida por esta comunidad de práctica como la crisis del campo de la educación en ciencias.

Ahora bien, las reformas curriculares en la enseñanza de las ciencias han comenzado a ser consideradas por los investigadores como una evidencia fuerte del desarrollo y la consolidación del campo de la educación en ciencias. En este sentido, se hace pertinente llevar a cabo una breve descripción histórica de la serie de reformas realizadas desde finales de 1800 hasta los actuales momentos. Para ello, se focaliza esta representación histórica en las transformaciones progresivas que han sufrido los propósitos de la enseñanza de las ciencias, y en la manera como estos fueron organizados, tanto en el currículum como en la instrucción. Adicionalmente, se tendrán en cuenta los diferentes modelos de enseñanza de las ciencias que están alineados con los propósitos específicos.
En este sentido, los investigadores consideran que los propósitos que han direccionado la configuración de la educación en ciencias en la escuela a través del currículum y la instrucción son los siguientes: (1) el conocimiento empírico de los sistemas físicos y biológicos (productos de las ciencias); (2) los métodos de investigación (procesos de las ciencias); y (3) el desarrollo personal del estudiante. Desde luego que estos han direccionado al interior de los grupos de investigación el diseño de los diferentes modelos de enseñanza que han venido siendo utilizados por los profesores en las aulas de ciencias desde esa época (Bybee, 1977).

Así pues, el primer propósito incluye el rango de observaciones acumuladas y la información sistemática acerca del Universo, es decir, en sus formas variadas, los hechos, los conceptos, las generalizaciones y los esquemas conceptuales generados por los científicos son parte de este propósito. Por tanto, cuando se diseña la enseñanza y el aprendizaje, el diseñador intenta responder el interrogante ¿qué conocen los científicos de una disciplina específica?

El segundo propósito abarca las técnicas de investigación o las prácticas científicas, las cuales se materializan a través de las competencias para resolver problemas. Por ejemplo, observar, predecir, formular hipótesis, argumentar, modelar, explicar, entre otras, son consideradas habilidades científicas. Adicionalmente a estas competencias, los hombres de ciencias deberían presentar actitudes como ser abiertos a la crítica, reconocer el error, mantener la curiosidad, ser honestos con la observación, predicción, formulación de hipótesis, modelación, recolección de datos, argumentación, y mantener un cuestionamiento permanente de los resultados. En consecuencia, los diseñadores curriculares intentan darle respuesta al interrogante ¿qué hacen los científicos para producir nuevo conocimiento?

Finalmente, el currículum y la instrucción deberían también tomar en consideración el desarrollo del estudiante. Este propósito incluye los requisitos intelectuales, emocionales, físicos y sociales necesarios para que el estudiante 
logre internalizar y desarrollar los productos y los procesos de las ciencias. Desde luego, estos elementos son comunes para todas las disciplinas del plan de estudios y deben de ser alineados con los antecedentes de los estudiantes singulares. El reconocimiento de este propósito es un intento de darle respuesta a la pregunta ¿qué puede el estudiante singular conocer y hacer en un nivel de desarrollo particular?

En consonancia con los anteriores presupuestos, estos propósitos fundamentales han direccionado el diseño del currículum y la instrucción de la educación en ciencias a lo largo de su historia. De hecho, durante la evolución de este campo se ha evidenciado que las necesidades propias de una región, junto con la serie de constructos novedosos producidos al interior de esta comunidad de práctica, han inducido a los diseñadores de las políticas educativas en ciencias a focalizar la enseñanza de estas disciplinas en uno de los tres propósitos mencionados. En efecto, este hecho produjo hasta el momento, en la educación en ciencias, una variedad de énfasis curriculares, los cuales quizás estarían alineados con los intereses de los gobiernos de turno.

Tomando como referencia el examen y la organización de los tres propósitos dentro del currículum de la educación en ciencias, se describirán brevemente las transformaciones históricas que este ha sufrido. Desde luego, esta situación probablemente serviría como una evidencia que apoyaría la tesis de que el campo de la educación en ciencias es un área que ha alcanzado una identidad de disciplina científica, de acuerdo con los criterios disciplinares de Toulmin (1972).

En coherencia con este presupuesto, los diferentes énfasis curriculares han estado fundados por los marcos que subyacen a las percepciones y los modelos de la enseñanza de las ciencias provenientes del campo en mención. Naturalmente, dichos modelos están determinados por el énfasis que hagan los diseñadores curriculares y educadores en uno de los propósitos de la enseñanza de las ciencias, con el fin de hacerle frente a las necesidades claves de la sociedad a la cual ellos pertenecen. Por ejemplo, según Bybee (1977), la combinación de la expansión industrial y la emigración desde las áreas rurales a finales de 1800 apoyaron la consolidación de dos modelos: uno sobre el estudio de la naturaleza, el cual tuvo como propósito central el desarrollo del sujeto, y el otro se centró en la comprensión del conocimiento donde el estudiante debería internalizar las teorías, los conceptos y los principios de la disciplina.

En los primeros años del siglo XX, la educación en ciencias de la escuela secundaria estuvo alineada con el propósito de que los estudiantes desarrollaran el conocimiento del contenido de las ciencias. Así, esta percepción generó el modelo de enseñanza de la ciencia fundamental. Este modelo fue propuesto por líderes educativos como Francis Parker, Wilbur Jackman, William Harris, y E. G. Howe, como una consecuencia de la transición de los países desde una sociedad agraria a una tecnológica-industrial. De hecho, estos personajes ejercieron una fuerte influencia en los gobernantes y diseñadores de políticas educativas de la época, con el fin de que sus propuestas fueran asumidas e implementadas en las escuelas.

Durante este momento histórico, algunos investigadores de la educación en ciencias ejercieron una contrafuerza al modelo centrado en el conocimiento disciplinar, moviéndose hacia una percepción focalizada en el desarrollo del sujeto y conocida como modelo de estudio de la naturaleza. Esta transformación curricular se debió a la migración de la población desde las áreas rurales a las urbanas, produciéndose una sobrepoblación de las últimas y, como consecuencia, una disminución de las posibilidades de empleo, situación que se vio reflejada en la crisis agrícola que sufrieron muchos países durante este tiempo (como EE. UU.). Asimismo, los investigadores y diseñadores de políticas educativas comenzaron a visualizar que la educación en ciencias podría ayudar a superar esta crisis, es decir, a motivar a las personas para que regresaran a sus actividades agrícolas. Para ello diseñaron programas curriculares cuya meta era brindar la oportunidad a la población para alcanzar un desarrollo personal y social, el cual les permitiría vivir una vida rica y feliz sin importar cuál fuera su profesión o negocio (Jenkins, 2000). 
Por otro lado, en la década de 1960, Dewey comenzó a sugerir que el currículum y la instrucción en ciencias se deberían focalizar en el desarrollo de las competencias científicas. Así pues, él consideró que la educación en ciencias tendría que ser funcional, centrada en el estudiante y reflejar las realidades de la época. Para ello, esta generaría los espacios en donde el estudiante, a través de las actividades de aprendizaje y las prácticas científicas, comenzaría a emular la resolución de problemas de orden social. Por lo general, los educadores de ciencias y los diseñadores curriculares, influenciados por esta filosofía pragmática, dirigieron reformas curriculares cuyo propósito primario fue el aprendizaje de los métodos de investigación de las ciencias.

Otro elemento que ejerció una fuerte influencia en las reformas curriculares focalizadas en el conocimiento y los métodos científicos fue la carrera espacial que sostuvo EE. UU. con la antigua Unión Soviética, donde el lanzamiento del Sputnik fue catalogado como el agente dinamizador de esta nueva percepción de la educación en ciencias. Quizás la "superioridad científica” de la Unión Soviética fue real o imaginaria, sin embargo, sirvió para que dicha reforma fuera desarrollada con mayor velocidad y amplitud. Naturalmente, la reforma producida en este tiempo adoptó como modelo de educación en ciencias el descrito por Jerome Bruner (1960) en el libro The Process of Education. En este se asume como propósito primario el conocimiento de la disciplina, y los métodos científicos el medio para lograrlo. Posteriormente, la NASTA, en el libro Theory Into Action, declaró como propósitos fundamentales los esquemas conceptuales y los procesos de las ciencias.

En cuanto a la década del 70, los investigadores comenzaron a mostrar un interés nuevamente por el propósito curricular que hace referencia al desarrollo del sujeto en aspectos como la identidad y la autoestima. Esta situación llevó a que los diseñadores de las políticas educativas en ciencias sugirieran que el currículum se configurara a partir de un equilibrio entre los tres propósitos previamente mencionados (conocimiento, métodos científicos y desarrollo del sujeto). Esta nueva postura curricular recibió el apoyo de eruditos como Bruner (1961) y Piaget (1971), quienes pusieron a disposición sus marcos teóricos de la psicología cognitiva y los métodos humanísticos para la enseñanza de las ciencias.

Por lo que se refiere a los movimientos de las reformas curriculares de la década de 1980 y comienzos de 1990, se afirma que los marcos teóricos sugeridos por las diferentes asociaciones del campo de la educación en ciencias han ejercido una fuerte influencia en el diseño de los currículos prescriptos, los cuales direccionan la educación en esta área en los EE. UU. a nivel estatal y local. Por ejemplo, instituciones como la American Association for the Advancement of Science (AAAS, 1989); la National Science Teachers Association (NSTA, 2003); la The National Center for Improving Science Education (NCISE), entre otras, durante este período financiaron proyectos tales como The Science for All Americans y la publicación de Benchmarks for Scientific Literacy; Scope, Sequence, and Coordination; y el proyecto the National Science Education Standards. Desde luego que la mayoría de estos proyectos fueron dirigidos por educadores de profesores e investigadores del campo de la educación con el propósito de impactar las aulas de ciencias de manera efectiva.

\section{CONCLUSIONES}

Las dos tradiciones del cambio conceptual y sociocultural que han venido configurando la investigación en el campo de la educación en ciencias, generan dos posturas en cuanto a la forma como se utilicen. En primer lugar, algunos investigadores pueden concebirlas como un todo que es más grande que la suma de las partes. Así pues, esta perspectiva los lleva a creer que la interacción sinérgica de las dos tradiciones proporciona a la comunidad de práctica una rica y profunda comprensión de las diferentes problemáticas sobre la enseñanza y el aprendizaje de las ciencias.

En segundo lugar, los miembros del campo de la educación en ciencias pueden considerar que cada una de las dos tradiciones actúa de manera independiente, asumiendo las problemáticas de 
la enseñanza y el aprendizaje de manera diferente. Esta situación podría generar en los miembros de cada tradición la idea de que las teorías y los métodos que subyacen a su tradición son más efectivos y eficientes que las otras, asumiendo que el todo es menos que la suma de las partes.

Las dos tradiciones presentan una serie de diferencias en cuanto a sus marcos teóricos y metodológicos que las sustentan, las cuales le dan su estatus epistemológico. Desde luego, cada una de estas sostiene sus concepciones acerca de la naturaleza y la función del conocimiento, además de los métodos de investigación apropiados para la construcción colegiada de este. Adicionalmente, en los miembros del campo de la educación en ciencias existe la creencia de que estas diferencias difícilmente podrán superarse como consecuencias de que los integrantes de estas comunidades de práctica poseen un sistema de conocimientos, creencias y valores que ayudan a continuar extendiendo y consolidando dichas perspectivas.

A través de sus estudios, las tradiciones del cambio conceptual y sociocultural han bridado intuición a los investigadores sobre el diálogo que llevan a cabo los estudiantes: en primer lugar, con la naturaleza y en segundo lugar con los demás miembros de la comunidad de práctica áulica. Así pues, dichas tradiciones por medio de sus estudios ilustran la manera como los estudiantes interactúan con los fenómenos naturales para construir una primera idea del funcionamiento de estos, la cual sirve como insumo que permite el establecimiento de la transacción de significados y formas de significar. Desde luego, esta última se convierte en un auténtico escenario sociocultural donde los estudiantes llegan a un consenso sobre el comportamiento del fenómeno natural estudiado.

Por otra parte, la investigación de la educación en ciencias en sus comienzos estuvo fundamentada por el marco teórico de las ciencias experimentales y de la psicología conductista, donde el control y la correlación de variables resultó ser un elemento clave para la validez y la confiabilidad de los resultados. De hecho, el propósito fundamental de estos estudios fue producir generalizaciones universales, en su mayoría descontextualizadas, de la misma forma a las generadas en las ciencias experimentales (proceso-producto). Desde luego, los investigadores pertenecientes a este paradigma se interesaron por estudiar los siguientes aspectos de la conducta docente en el aula de clase: respuestas a las situaciones de indisciplina, asignación de turnos para hablar, establecimientos de reglas de juego, utilización de preguntas fáciles o difíciles, frecuencias de elogios o críticas, tiempo de espera, entre otras. Para ello, observaron las acciones tanto de los enseñantes como de los aprendices a través de instrumentos con escalas de observación categorizadas, casi siempre del tipo de "baja inferencia" (aquellas que dan cuenta de las situaciones observadas, en vez de juzgar o evaluar la calidad de las actividades observadas, lo que sería considerado de "alta inferencia” [Shulman, 1987]). Adicionalmente, las unidades de análisis son el día de la clase, las acciones del profesor y de los estudiantes.

Ahora bien, en épocas recientes, los marcos metodológicos que subyacen a los estudios en este campo han cambiado hacia una metodología de corte cualitativo e interpretativo, la cual se ha apoyado para su desarrollo en los marcos teóricos de disciplinas como la antropología, la sociología y la lingüística. De ahí que para esta especie de metodología hermenéutica, la subjetividad y la narrativa son las herramientas claves para poder comprender las situaciones singulares del objeto-sujeto bajo estudio. Asimismo, el propósito de estas investigaciones es el de producir generalizaciones naturalistas, las cuales sirven para interpretar las acciones inteligentes de los diferentes miembros del sistema escolar dentro de un contexto particular.

Así, dentro de este paradigma de investigación interpretativo, los estudios van desde el microanálisis de las interacciones tanto verbales como no verbales en el aula de clase durante la enseñanza de un tópico específico, hasta el macroanálisis de toda una escuela secundaria. Para ello, el investigador utiliza herramientas metodológicas como observación participante, estimulación del recuerdo, pensamiento en voz alta, notas de campo, entre otras. Hay que 
mencionar, además, que para darle confiabilidad $\mathrm{y}$ validez a los asertos que se generen se debe tener en cuenta el proceso de la triangulación por fuente, investigador y método de estudio (Denzin, 1978).

Finalmente, en la última década, las investigaciones en educación en ciencias vienen utilizando una perspectiva de carácter mixto (cuantitativocualitativo). Probablemente esta situación se ha generado como consecuencia de que este enfoque permite llevar a cabo la triangulación, dándole confiabilidad y validez a la serie de resultados proveniente del estudio en cuestión.

\section{REFERENCIAS BIBLIOGRÁFICAS}

Abell, S. K. y Lederman, N. G. (Eds.). (2007). Handbook of Research on Science Education. Mahwah, NJ: Lawrence Erlbaum.

Adey, P. y Shayer, M. (1993). An Exploration of Long-Term Far-Transfer Effects Following an Extended Intervention Program in the High School Science Curriculum. Cognition and Instruction, 11(1), 1-29.

American Association for the Advancement of Science. (1989). Project 2061: Science for all Americans. Washington, DC: American Association for the Advancement of Science.

Anderson, C. W. (2007). Perspectives on science learning. En S. K. Abell y N. G. Lederman (Eds.), Handbook of research on science education (pp. 3-30). NY: Routledge.

Ausubel, D., Novak, J. y Hanesian, M. (2005). Psicología educativa: un punto de vista cognoscitivo. México: Trillas.

Bandiera, M., Caravita, S., Torracca, E. y Vicentini, M. (1999). Research in Science Education in Europe. Netherlands: Springer Science Business Media Dordrecht.

Boenig, R. W. (1969). Research in science education, 1938 through 1947. New York: Teachers College Press.

Brown, J., Collins, A. y Duit, P. (1989). Situated Cognition and the Culture of Learning. Educational Researcher, 18(1), 32-42.

Bruner, J. (1961). The act of discovery. Harvard Educational Review, 30, 21-32.

Bruner, J. (1960). The Process of Education. New York: Vintage Books.
Bybee, R. W. (1977). The New Transformation of Science Education. Science Education, 61(1), 85-97.

Carey, S. (1985). Conceptual change in childhood. Cambridge, MA: Bradford Books, MIT Press.

Chinn, C. y Brewer, W. (1993). The Role of Anomalous Data in Knowledge Acquisition: A Theoretical Framework and Implications for Science Instruction. Review of Educational Research, 63(1), 1-49.

Collins, A., Brown, J. S. y Newman, S. E. (1989). Cognitive apprenticeship: Teaching the crafts of reading, writing, and mathematics. En L. B. Resnick (Ed.), Knowing, learning, and instruction: Essays in honor of Robert Glaser (pp. 453-494). Hillsdale, NJ: Lawrence Erlbaum.

Denzin, N. (1978). The Research Act in Sociology: A Theoretical Introduction to Sociological Methods. London: Butterworths.

Driver, R. (1983). The Pupil as Scientist. Philadelphia: The open University Press.

Driver, R. (1973). The Representation of Conceptual Frameworks in young adolescent science students (Unpub. Ph. D. thesis). University of Illinois, Urbana, Illinois.

Duit, R. (2007). Science education research internationally: Conceptions, research methods, domains of research. Eurasia Journal of Mathematics, Science \& Technology Education, 3(1), 3-15.

Duit, R., Gropengießer, H. y Kattmann, U. (2005). Towards science education research that is relevant for improving practice: The model of educational reconstruction. En H. E. Fischer, (Ed.), Developing standards in research on science education (pp. 1-9). London: Taylor \& Francis.

Duit, R. y Treagust, D. (1998) Learning in science - From behaviourism towards social constructivism and beyond. En B. Fraser y K. Tobin (Eds.), International handbook of science education (pp. 3-26). Dordrecht, The Netherlands: Kluwer Academic Publishers.

Fraser, B. J., Tobin, K. G. y McRoobbie, C. J. (Eds.). (2012). Second International Handbook of Science Education. London New York: Springer.

Fraser, B. J. y Tobin, K. G. (Eds.). (1998). International Handbook of Science Education. Dordrecht, The Netherlands: Kluwer. 
Gabel, D. (Ed.). (1994). Handbook of Research on Science Teaching and Learning. Nueva York: MacMillan.

Gorard, S. y Taylor, C. (2004). Combining Methods in Educational and Social Research. The promise of design studies. New York: McGraw-Hill Education.

Hennessy, S. (1993). Situated Cognition and Cognitive Apprenticeship: Implications for Classroom Learning. Studies in Science Education, 22(1), 1-41.

Jenkins, E. W. (2000). Research in Science Education: Time for a Health Check? Studies in Science Education, 35, 1-26.

Karplus, R. (1980). Teaching for The Development of Reasoning. Research in Science Education, 10, 1-10.

Knorr-Cetina, K. D. (1981). The Manufacture of Knowledge: An Essay on the Constructivist and Contextual Nature of Science. New York Oxford: Pergamon Press.

Kuhn, T. S. (1970). The structure of scientific revolutions. Chicago: The University of Chicago Press.

Lawson, A. y Thompson, L. (1988). Formal reasoning ability and misconceptions concerning genetics and natural selection. Journal of Research in Science Teaching, 25(9), 733-746.

Lemke, J. L. (1990). Talking science: Language, learning, and values. Norwood, NJ: Ablex.

Martinez-Vélez, A. (2013). Emergencias de cambio: entre el modelo pedagógico tradicional y la necesidad de aprendizajes significativos. Praxis, 9, 73-82.

McGinn, M., Roth, W., Boutonné, S. y Woszczyna, C. (1995). The transformation of individual and collective knowledge in elementary science classrooms that are organised as knowledge-building communities. Research in Science Education, 25(2), 163-189.

National Science Teachers Association (2003). Standards for science teacher preparation. Washington, DC: National Science Teachers Association. Recuperado de https://digitalcommons.unl.edu/cgi/viewcontent. cgi?referer $=$ https://scholar.google.es/\&httpsred ir $=1$ \&article $=1085 \&$ context $=$ teachlearnfacpub

Novak, J. D. (1987). Human Constructivism: Toward a Unity of Psychological and
Epistemological Meaning Making. En J. D. Novak (Ed.), Proceedings of the Second International Seminar on Misconceptions and Educational Strategies in Science and Mathematics (pp. 349-360). New York: Departament of de Education, University of Cornell.

Osborne, R. y Freyberg, P. (1985). Learning in Science: the implications of Children's Science. London: Heinemann Publishers.

Piaget, J. (1971). Genetic Epistemology. New York: Norton.

Posner, G., Strike, K., Hewson, P. y Gertzog, W. (1982). Accommodation of a scientific conception: Toward a theory of conceptual change. Science Education, 66(2), 211-227.

Psillos, D. (1997). Teaching introductory electricity. En A. Tiberghien, E.-L. Jossem y J. Barojas (eds.), Connecting Research in Physics Education with Teacher Education. Recuperado de http://www.physics.ohio-state. edu/ jossem/ICPE/BOOKS.html

Rogoff, B. (1990). Apprenticeships in thinking: Cognitive development in social context. New York: Oxford University Press.

Roth, W. M. (1995). Teacher questioning in an open-inquiry learning environment: interactions of context, content and student responses. Journal of Research in Science Teaching, 33, 709-736.

Roth, W. M. (2001). Situating cognition. Journal of the learning sciences, 10, 27-61.

Shayer, M. y Wylam, H. (1981). The development of the concepts of heat and temperature in 10-13 year-olds. Journal of Research in Science Teaching, 18(5), 419-434.

Shulman, L. (1987). Knowledge and Teaching. "Foundations of the New Reform". Harvard Educational Review, 57(1), 1-21.

Sutton, C. (1992). Words, Science and Learning. London: Open University Press.

Toulmin, S. (1972). Human understanding: the collective use and evaluation of concepts. Princeton: University Press Princeton.

Viennot, L. (1979). Spontaneous Reasoning in Elementary Dynamics. European Journal of Science Education, 1(2), 205-221.

von Glasersfeld, E. (1995). Radical Constructivism: A Way of Knowing and Learning. London Washington, D. C.: The Falmer Press. 
Vosniadou, S. (1994). Capturing and modeling the process of conceptual change. Learning and Instruction, 4(1), 45-69.
Vygotsky, L. S. (1986). Thought and language. London MA: MIT Press. 